\title{
Psicooncología
}

ISSN: 1696-7240

\section{El consumo de alcohol en los supervivientes al cáncer: estado de la cues- tión y propuestas para el desarrollo de intervenciones psicológicas}

\author{
Francisco García-Torres ${ }^{1}$; Francisco J. Alós ${ }^{2}$; Rosario Castillo-Mayén ${ }^{3}$
}

Recibido: 6 de junio de 2017 / Aceptado: 23 de enero de 2018

Resumen: Objetivo: El consumo de alcohol se ha establecido como un factor de riesgo para el desarrollo del cáncer de forma consistente a lo largo de los años. En los supervivientes al cáncer el consumo de alcohol después del tratamiento alcanza valores significativos y además el alcohol se ha asociado con aspectos relevantes de la supervivencia, como una mayor depresión y una peor calidad de vida. Sin embargo, no hay una gran variedad de intervenciones cuyo objetivo sea reducir el consumo de alcohol en este grupo de pacientes. Método: Se llevó a cabo una revisión sobre el consumo de alcohol en los pacientes y supervivientes al cáncer y las intervenciones psicológicas disponibles para reducir el consumo, en los últimos 10 años, del año 2007 al 2017. Las bases de datos consultadas fueron PubMed, Web of Science (WOS) y PsycInfo. Se utilizaron los siguientes descriptores: alcohol, cancer patients, survivors, psychological treatment. Resultados: no se observan intervenciones psicológicas diseñadas específicamente para reducir el consumo de alcohol en los supervivientes al cáncer en la literatura previa. La mayoría tienen como objetivo el aumento de las conductas de salud utilizando intervenciones de tipo psicosocial o proporcionando información sobre el alcohol. Solamente una intervención mostró resultados significativos para reducir el consumo de alcohol, en una intervención diseñada para reducir el peso en supervivientes al cáncer de mama. Conclusiones: la relevancia del consumo de alcohol en este grupo de pacientes sugiere la necesidad de que se desarrollen una mayor variedad de intervenciones psicológicas que tengan como objetivo específico la reducción del consumo de alcohol en los pacientes que han superado la enfermedad.

Palabras clave: Cáncer; oncología; supervivientes; alcohol; intervenciones psicológicas.

\section{[en] Alcohol consumption in cancer survivors: state of the question and proposals for the development of psychological interventions}

\footnotetext{
Abstract: Objective: Alcohol consumption has been established as a risk factor for cancer development consistently over the years. In cancer survivors alcohol consumption after treatment achieves significant values, and furthermore alcohol has been associated with relevant aspects of survival, such

1 Francisco García-Torres. Departamento de Psicología de la Universidad de Córdoba / Instituto Maimónides de Investigación Biomédica de Córdoba (IMIBIC) / Hospital Universitario Reina Sofía.

E-mail: z12gatof@uco.es

2 Francisco J. Alós. Departamento de Psicología de la Universidad de Córdoba / Instituto Maimónides de Investigación Biomédica de Córdoba (IMIBIC) / Hospital Universitario Reina Sofía.

E-mail: ed1alcif@uco.es

3 Rosario Castillo-Mayén. Departamento de Psicología de la Universidad de Córdoba / Instituto Maimónides de Investigación Biomédica de Córdoba (IMIBIC) / Hospital Universitario Reina Sofía.

E-mail: z92camam@uco.es

* Dirección de correspondencia: Francisco García-Torres. Departamento de Psicología de la Universidad de Córdoba / Instituto Maimónides de Investigación Biomédica de córdoba (IMIBIC) / Hospital Universitario Reina Sofía. Avda San Alberto Magno s/n C.P. 14071. Córdoba. España. E-mail: z12gatof@uco.es
} 
as a high depression and a lower quality of life. There is no a great variety of interventions, however, whose objective be reduce alcohol consumption in this group of patients. Method: A review of the alcohol consumption in cancer patients and survivors and the available psychological interventions to reduce consumption in the last 10 years were carried out, from 2007 to 2017. The consulted databases were PubMed, Web of Science (WOS) and PsycInfo. The following descriptors were used: alcohol, cancer patients, survivors, psychological treatment. Results: Psychological interventions specifically designed to reduce alcohol consumption in cancer survivors are not observed in previous literature. Most of them focused in enhance health behaviors using psychosocial interventions or information about alcohol. Only one intervention showed significant results to reduce alcohol consumption in an intervention designed to reduce weight in breast cancer survivors. Conclusions: the relevance of alcohol consumption in this patients group suggest the need of develop a greater variability of psychological interventions that aim to control alcohol consumption in patients who have overcome the disease.

Keywords: Cancer; oncology; survivors; alcohol; psychological interventions.

Sumario. 1. Introducción 2. Método 3. Discusión 4. Conclusiones 5. Referencias bibliográficas.

Cómo citar: García-Torres F, Alós FJ, Castillo-Mayén R. El consumo de alcohol en los supervivientes al cáncer: estado de la cuestión y propuestas para el desarrollo de intervenciones psicológicas. Psicooncología 2018;15:65-73. Doi: 10.5209/PSIC.59175.

\section{Introducción}

El uso nocivo del alcohol es un problema de salud pública relevante a nivel mundial. Según la Organización Mundial de la Salud, un 5,9\% de las muertes a nivel global se producen por esta causa, alcanzando el $25 \%$ de la mortalidad de la población mundial que está entre los 20 a 39 años. Además, el consumo elevado de alcohol se asocia consistentemente con una gran variedad de enfermedades tanto comportamentales como físicas ${ }^{(1)}$. En este sentido, existe evidencia que apoya la afirmación de que el consumo de alcohol es un factor de riesgo muy relevante para el desarrollo del cáncer, observándose esta relación en la mayoría de los tipos de cáncer, incluyendo no solamente aquellos cuya localización podría sugerir una mayor exposición, como por ejemplo el cáncer de cabeza y cuello y el cáncer oral, sino en otros tipos de cáncer como el de mama y el colorrectal. Es necesario destacar que en los pacientes se observa cierto desconocimiento acerca de esta relación, y esta circunstancia puede empeorar el desarrollo de la enfermedad afectando muy negativamente a la supervivencia a la misma ${ }^{(2-7)}$.

Sin embargo, existe evidencia de que el consumo de alcohol no aumenta el riesgo de recurrencia a la enfermedad ni tampoco se asocia con una mayor mortalidad en este grupo de pacientes ${ }^{(8)}$ aunque, en otro sentido, diferentes autores apoyan la idea de que en aquellos pacientes que han superado un cáncer primario y siguen consumiendo alcohol, el riesgo de desarrollo de un segundo cáncer es más elevado y esta relación se observa, a semejanza de lo comentado anteriormente, en la mayoría de los tipos de cáncer, incluyendo el cáncer de cabeza y cuello y el de mama ${ }^{(9-14)}$. Es necesario tener en cuenta que los efectos perniciosos del consumo de alcohol parecen depender de la cantidad consumida. Por ejemplo, se ha observado en supervivientes al cáncer de mama con un elevado consumo de alcohol, una mayor recurrencia de la enfermedad y una mayor mortalidad asociada, sobre todo en mujeres 
postmenopáusicas y que además tienen sobrepeso ${ }^{(15,16)}$. Por otra parte, el consumo de alcohol de forma moderada, en concreto de vino, puede aumentar la supervivencia al cáncer, como se ha observado en supervivientes al linfoma de tipo no Hogdkin, lo que puede explicarse por el efecto positivo de los polifenoles abundantes en el vino, cuyos efectos anti-cáncer están bien establecidos en estudios de laboratorio ${ }^{(17)}$.

La relevancia del estudio del alcoholismo en los pacientes que han superado un cáncer queda explícita cuando se observa que un porcentaje elevado de los mismos, hasta el $40 \%$, manifiestan continuar tomando alcohol después de finalizar el tratamiento activo, y además el consumo elevado de alcohol aparece en un tercio del total de supervivientes, particularmente en las pacientes que han superado el cáncer de cuello de útero y en supervivientes a múltiples diagnósticos de cáncer, en los que se observan un mayor consumo de alcohol y una mayor presencia de conductas poco saludables en términos de tabaquismo y una peor alimentación ${ }^{(18-25)}$. Por otro lado, se observan menores niveles en el consumo de alcohol en pacientes que han superado el cáncer colorrectal, gástrico y de mama, especialmente si además padecen hipertensión ${ }^{(26-28)}$.

El consumo de alcohol en los supervivientes al cáncer se asocia además con otros aspectos relevantes relacionados con el bienestar psicológico de los supervivientes. En este sentido, diferentes autores aportan datos que apoyan la idea de que el consumo de alcohol en supervivientes a diferentes tipos de cáncer, como el oral y el cáncer de cabeza y cuello, se asocia consistentemente con la depresión y una peor calidad de $\operatorname{vida}^{(29,30)}$. Además, ser hombre, una menor edad, un nivel socioeconómico elevado, y el consumo de tabaco se asocian consistentemente con el consumo elevado de alcohol después del tratamiento ${ }^{(28,31-33)}$.

Sin embargo, también se observa que una vez finalizado el tratamiento aumenta significativamente la presencia de conductas encaminadas a mejorar la salud, lo que frecuentemente conlleva una reducción del consumo de alcohol ${ }^{(28,34-38)}$, aunque por otro lado se ha observado que los supervivientes al cáncer no tienen una elevada adherencia a las recomendaciones para llevar una dieta saludable, incluyendo el consumo de alcohol cuando se comparan con individuos sin una historia previa de cáncer $^{(39)}$, lo que puede estar relacionado con la falta de comprensión por parte del paciente de las relaciones que existen entre el consumo de alcohol y la aparición del cáncer. Además se observa que en determinados tipos de pacientes, como por ejemplo en los supervivientes al cáncer colorrectal, existe una falta de recomendaciones específicas para reducir el consumo de alcohol, ya que un porcentaje significativo de los mismos, alrededor del 15\%, nunca han recibido instrucciones en este sentido ${ }^{(40)}$.

A la luz de los resultados comentados previamente, parece relevante que existan intervenciones cuyo objetivo sea reducir el consumo de alcohol en los supervivientes al cáncer, debido a su relevancia clínica. Por lo tanto, los objetivos de la presente revisión es realizar una búsqueda de las diferentes intervenciones desarrolladas para reducir el consumo de alcohol en los supervivientes al cáncer y analizar, llegado el caso, su eficacia.

\section{Método}

Se llevó a cabo una revisión de las diferentes intervenciones desarrolladas para reducir el consumo de alcohol en los supervivientes al cáncer, utilizando las bases de datos 


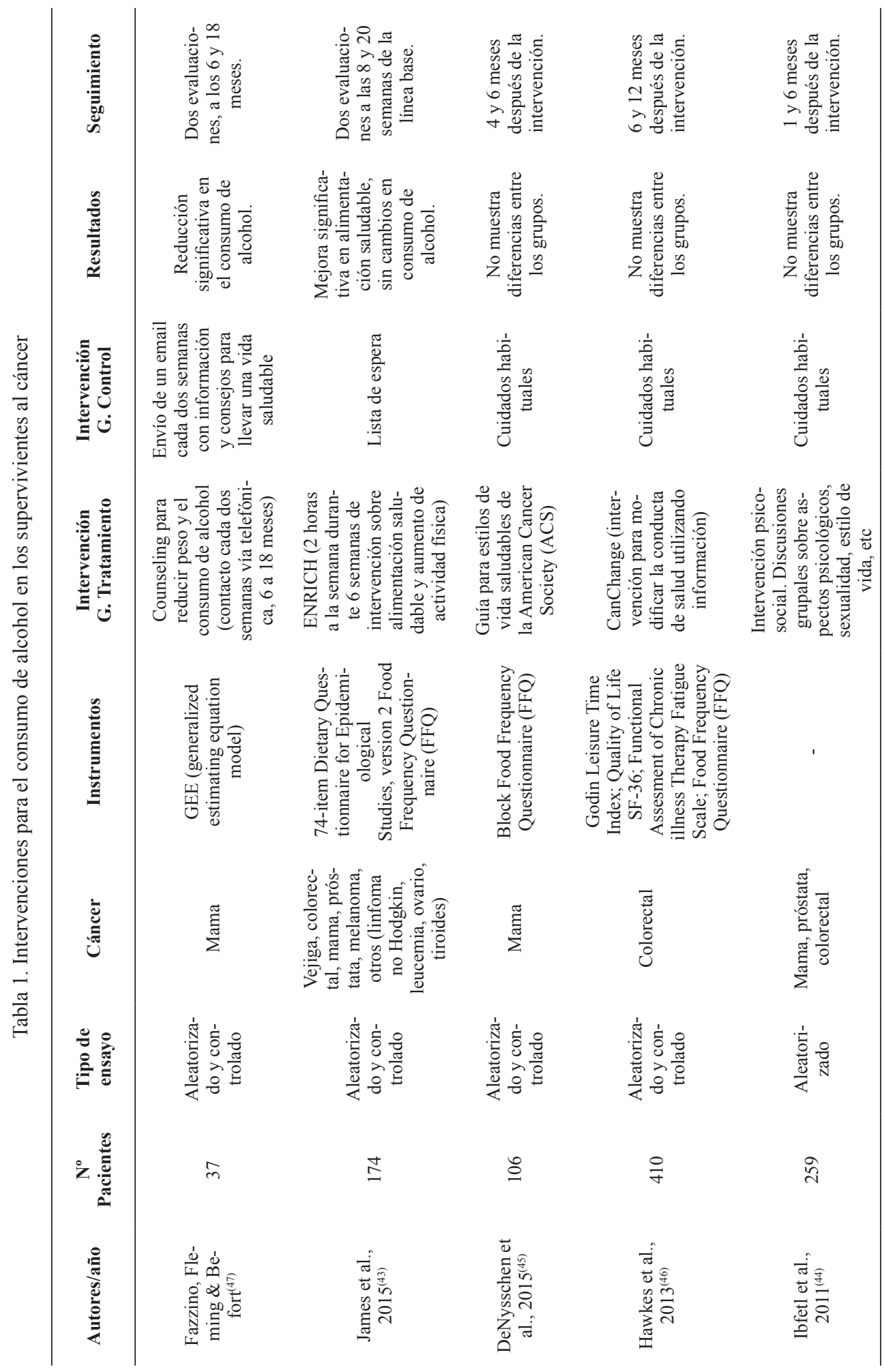


PubMed, Web of Science (WOS) y PsycInfo. Se delimitó el periodo de búsqueda a los 10 últimos años, del 2007 al 2017, incluyendo en los resultados examinados los abstract y cuando estaban disponibles, los artículos completos. Además del periodo temporal se tuvieron en cuenta los artículos cuyos participantes eran mayores de 18 años y habían finalizado el tratamiento activo de la enfermedad, con los siguientes descriptores: alcohol, cancer patients, survivors, psychological treatment. En total se encontraron cinco artículos que habían llevado a cabo intervenciones para reducir el consumo de alcohol en los supervivientes, dentro de diferentes estrategias para promover un estilo de vida saludable. Una descripción de las intervenciones encontradas puede consultarse en la Tabla 1.

\section{Discusión}

El consumo de alcohol se ha establecido como un factor de riesgo muy relevante para el desarrollo del cáncer, no solamente para aquellos tipos de cáncer que se desarrollan en áreas próximas a la función digestiva ${ }^{(9-14,41)}$. Además de aspectos relacionados con la aparición de la enfermedad en los supervivientes, el consumo de alcohol se relaciona con la depresión y una peor calidad de vida ${ }^{(29,30)}$. A pesar de esta circunstancia, no se observa en la literatura previa a este respecto una gran variedad de intervenciones que hayan sido diseñadas con el objetivo de reducir el consumo de alcohol en los supervivientes. La mayoría de las intervenciones se incluyen dentro de otras estrategias para promover un estilo de vida saludable, no apareciendo ensayos que tengan como objetivo específico la reducción del consumo de alcohol en este grupo de pacientes. Analizando los datos obtenidos por diferentes autores, solamente se observan reducciones significativas en el consumo de alcohol en uno de los estudios analizados, que incluye una intervención más amplia dirigida a promover la pérdida de peso en un grupo de supervivientes al cáncer de mama ${ }^{(43-47)}$. A pesar de estos resultados, el limitado tamaño muestral del grupo que recibió la intervención y la inclusión del consumo de alcohol como elemento secundario dentro de los objetivos de la intervención, nos lleva a tomar estos resultados positivos con cautela. En este sentido, hay algunos elementos que aparecen como relevantes en el momento de desarrollar una intervención psicológica que tenga como objetivo la reducción del consumo de alcohol en este grupo de pacientes. En primer lugar, el consumo de alcohol, aunque se observa en un porcentaje elevado de supervivientes a distintos tipos de cáncer, es particularmente elevado en supervivientes al cáncer de cuello uterino y en pacientes con múltiples diagnósticos de cáncer ${ }^{(18-25)}$, lo que resalta una vez más, la necesidad de llevar a cabo intervenciones para reducir el consumo de alcohol en estos grupos de pacientes. Además, existe poca concienciación en los pacientes acerca de las relaciones que existen entre el alcohol y el desarrollo del cáncer y un segundo cáncer primario, y las consecuencias negativas en términos de una peor calidad de vida y mayor depresión. En población general, se han aplicado diferentes técnicas psicológicas para aumentar la motivación por el cambio, como por ejemplo al automonitoreo del consumo, el análisis de las situaciones en las que se produce y el entrenamiento en estrategias de afrontamiento efectivas, que aparecen como eficaces, sobre todo cuando se implica a los familiares utilizando técnicas de mejora en las habilidades de comunicación y de evitación de conflictos, elementos que pueden tomarse como base para el desarrollo de intervenciones psicológicas cuyo objetivo sea reducir el consumo de alcohol en los supervivientes al cáncer ${ }^{(48,49)}$. Finalmente, para 
la evaluación tanto del consumo de alcohol como para evaluar la posible eficacia de una intervención que tenga como objetivo la reducción del consumo, podría utilizarse el instrumento desarrollado por el NCI, Food Frequency Questionnaire (FFQ) que cuenta con una adaptación al castellano ${ }^{(50)}$. Estas recomendaciones han de tomarse con la debida cautela, ya que la verdadera eficacia de una intervención ha de ponerse a prueba en ensayos con los adecuados niveles de control.

\section{Conclusiones}

El consumo de alcohol en los supervivientes al cáncer es un hecho relevante que merece una mayor atención. Sin embargo, las intervenciones desarrolladas hasta el momento no ofrecen unos resultados que avalen su eficacia. Para futuras intervenciones se recomienda incluir como elementos a tener en cuenta diferentes técnicas psicológicas para reducir el consumo de alcohol, como por ejemplo el entrenamiento en estrategias de afrontamiento y la mejora en las habilidades de comunicación.

\section{Referencias bibliográficas}

1. Organización Mundial de la Salud. Global status report on alcohol and health 2014. Suiza, Ginebra: OMS.

2. Boyle T, Fritschi L, Tabatabaei SM., Ringwald K, Heyworth, JS. Smoking, alcohol, diabetes, obesity, socioeconomic status, and the risk of colorectal cancer in a populationbased case-control study. Cancer Causes Control 2014;25: 1659-68. doi: 10.1007/s10552014-0470-7

3. Crozier E, Sumer, BD. Head and neck cancer. Med Clin North Am 2010; 94: 1031-46. doi. 10.1016/j.mcna.2010.05.014

4. Dumalaon-Canaria JA, Hutchinson AD, Prichard I, Wilson C. What causes breast cancer? A systematic review of causal attributions among breast cancer survivors and how these compare to expert-endorsed risk factors. Cancer Causes Control 2014; 25: 771-85. doi: 10.1007/s10552-014-0377-3.

5. Huber MA, Tantiwongkosi B. Oral and oropharyngeal cancer. Medical Clin North Am 2014; 98: 1299-321. doi: 10.1016/j.mena.2014.08.005.

6. Ligibel J. Lifestyle factors in cancer survivorship. J Clin Oncol 2012; 30: 3697-704.

7. Thompson CA. Diet and breast cancer: understanding risks and benefits. Nutr Clin Pract 2012; 27: 636-50. doi: 10.1200/JCO.2012.42.0638

8. Kwan ML, Chen WY, Flatt SW, Weltzien EK, Nechuta SJ, Poole EM, et al. Postdiagnosis alcohol consumption and breast cancer prognosis in the after breast cancer pooling project. Cancer Epidemiol Biomarkers Prevention 2013; 22: 32-41. doi: 10.1158/10559965

9. Adhikari J, Sharma P, Bhatt VR. Risk for secondary solid malignances after allogenic hematopoietic stem cell transplantation and preventive strategies. Future Oncol 2015; 11: 3175-85. doi: 10.2217/fon.15.252.

10. Lee DH, Roh JL, Baek S, Jung JH, Choi SH, Nam SY. et al. Second cancer incidence, risk factor, and specific mortality in head and neck squamous cell carcinoma. Otolaryngol Head Neck Surg 2013; 149: 579-86. doi: 10.1177/0194599813496373 
11. Li CI, Daling JR, Porter PL, Tang MT, Malone KE. Relationship between potentially modifiable lifestyle factors and risk of second primary contralateral breast cancer among women diagnosed with estrogen receptor-positive invasive breast cancer. J Clin Oncol 2009; 27:5312-18. doi: 10.1200/JCO.2009.23.1597

12. McLaughlin VH, Trentham-Dietz A, Hampton JM, Newcomb PA, Sprague BL. Lifestyle factors and the risk of a second breast cancer after carcinoma in situ. Cancer Epidemiol Biomarkers Prev 2014; 23:450-60. doi: 10.1158/1055-9965.EPI-13-0899

13. Tabuchi T, Ozaki K, Ioka A, Miyashiro I. Joint and independent effect of alcohol and tobacco use on the risk of subsequent cancer incidence among cancer survivors: A cohort study using cancer registries. Int J Cancer 2015; 137:2114-23. doi: 10.1002/ijc.29575

14. Travis LB, Demark-Wahnefried W, Allan JM, Wood ME, Ng AK. Aetiology, genetics and prevention of secondary neoplasms in adult cancer survivors. Nat Rev Clin Oncol 2013; 10: 289-301. doi: 10.1038/nrclinonc.2013.41

15. Kwan ML, Kushi LH, Weltzien E, Tam EK, Castillo A, Sweeney C. et al. Alcohol consumption and breast cancer recurrence and survival among women with early-stage breast cancer: the life after cancer epidemiology study. J Clin Oncol 2010; 28: 4410-6. doi: 10.1200/JCO.2010.29.2730

16. Weaver AM, McCann SE, Nie JN, Edge SP, Nochajski TH, Russell M, et al. Alcohol intake over the life course and breast cancer survival in Western New York Exposures and breast cancer (WEB) study: quantity and intensity of intake. Breast Cancer Res Treat 2013; 139: 245-53. doi: 10.1007/s10549-013-2533-y

17. Han X, Xheng T, Foss FM, Ma S, Holford TR, Boyle P, et al. Alcohol consumption and non-Hodgkin lymphoma survival. J Cancer Surviv 2010; 4: 101-9. doi: 10.1007/s11764009-0111-4

18. Saquib N, Pierce JP, Saquib J, Flatt SW, Natarajan L, Bardwell WA. et al. Poor physical health predicts time to additional breast cancer events and mortality in breast cancer survivors. Psychooncology 2011; 20: 252-59. doi: 10.1002/pon.1742

19. Funk GF, Karnell LH, Christensen AJ. Long-term health-related quality of life in survivors of head and neck cancer. Arch Otolaryngol Head Neck Surg 2012; 138: 123-33. doi: 10.1001/archoto.2011.234.

20. Krebs P, Coups EJ, Feinstein MB, Burkhalter JE, Steingart RM, Loque A. et al. Health behaviors of early-stage non-small cell lung cancer survivors. J Cancer Surviv 2012; 6: 37-44. doi: 10.1007/s11764-011-0191-9

21. Rausch SM, Millay S, Scott C, Pruthi S, Clark MM, Patten C, et al. Health behaviors among cancer survivors receiving screening mammography. Am J Clin Oncol 2012; 35: 22-31. doi: 10.1097/COC.0b013e318200598e

22. Reilley MJ, Jacobs LA, Vaughn DJ, Palmer SC. Health behaviors among testicular cancer survivors. J Community Support Oncol 2014; 12: 121-28. doi: 10.12788/jcso.0033.

23. Shinn EH, Swartz RJ, Thornton BB, Spiess PE, Pisters LL, Basen-Enqquist KM. Testis cancer survivors' health behaviors: comparison with age-matched relative and demographically matched population controls. J Clin Oncol 2010; 128: 2274-9. doi: 10.1200/JCO.2009.23.9608

24. Schootman M, Deshpande AD, Lynskey MT, Pruitt SL, Lian M, Jeffe DB. Alcohol outlet availability and excessive alcohol consumption in breast cancer survivors. J Prim Care Community Health 2013; 4:50-8. doi: 10.1177/2150131912443133

25. Burris JL, Andrykowski MA. Physical and mental health status and health behaviors of survivors of multiple cancers: a national, population-based study. Ann Behav Med 2013; 42: 304-12. doi: 10.1007/s12160-011-9290-0 
26. Jeong JR, Kim S, Jo SR, Joh JY, Kim YP. Health Behaviors of breast cancer survivors with hypertension: A propensity analysis of KNHANES III-IV (2005-2012). Plos One 2015; 10: e127346. doi: 10.1371/journal.pone.0127346.

27. Jo SR, Joh JY, Jeong JR, Kim S, Kim YP. Health behaviors of Korean gastric cancer survivors with hypertension: A propensity analysis of KNHANES III-IV (2005-2012). PloS One 2015; 10: e0126927. doi: 10.1371/journal.pone.0126927

28. Park B, Kong SY, Kim J, Park IH, Jung SY, Lee ES. Health behaviors of cancer survivors in nationwide cross-sectional survey in Korea: Higher alcohol drinking, lower smoking, and physical inactivity pattern in survivors with higher house hold income. Medicine 2015; 94: e1214. doi: 10.4143/crt.2017.129

29. Chen SC, Huang BS, Lin CY. Depression and predictors in Taiwanese survivors with oral cancer. Asian Pac J Cancer Prev 2013; 14: 4571-6. doi: 10.7314/APJCP.2013.14.8.4571

30. So WK, Chan RJ, Chan DN, Hughes BG, Chair SY, Choi KC, et al. Quality of life among head and neck cancer survivors at one year after treatment-a systematic review. Eur J Cancer 2012; 48(15): 2391-408. doi: 10.1007/s00405-016-3924-6

31. Schiller U, Inhestern J, Burger U, Singer S, Guntinas-Lichius O. Predictors of posttreatment smoking and drinking behavior of head and neck cancer survivors. Eur Arch Otorhinolaryngol 2016; 273: 3337-45.

32. Kim H, Kim MH, Park YS, Shin JY, Song YM. Factors that predict persistent smoking of cancer survivors. J Korean Med Sci 2015; 30: 853-9. doi: 10.3346/jkms.2015.30.7.853

33. Westmaas JL, Alcaraz KL, Berg CJ, Stein KD. Prevalence and correlates of smoking and cessation-relasted behavior among survivors of ten cancers: findings from a nationwide survey nine years after diagnosis. Cancer Epidemiol Biomarkers Prev 2014; 23: 1783 92. doi: $10.1038 /$ bjc. 2012.238

34. Kanera IM, Bolman CA, Mesters I, Willems RA, Beaulen AA, Lechner L. Prevalence and correlates of healthy lifestyle behaviors among early cancer survivors. BMC Cancer 2016; 16:4. doi: 10.1186/s12885-015-2019-x

35. Karlsen RV, Bidstrup PE, Christensen J, Larsen SB, Tjønneland A, Dalton SO, et al. Men with cancer change their health behavior: a prospective study from the Danish diet, cancer and healthy study. Br J Cancer 2012; 107: 201-6.

36. Moon SH, Lee DT, Son Y. Adherence to health-related lifestyle behavior recommendations and association with quality of life among cancer survivors and age-matched controls in Korea. Asian Pac J Cancer Prev 2013; 14: 2949-54. doi: 10.7314/APJCP.2013.14.5.2949

37. Vance V, Campbell S, McCargar L, Mourtzakis M, Hanning R. Dietary changes and food intake in the first year after breast treatment. Appl Physiol Nutr Metabol 2014; 39: 70714. doi: 10.1139/apnm-2013-0400

38. Williams K, Steptoe A, Wardle J. Is a cancer diagnosis a trigger for health behavior change? Findings from a prospective, population-based study. Br J Cancer 2013; 108 : 2407-12. doi: 10.1038/bjc.2013.254.

39. Zhang FF, Liu S, John EM, Must A, Demark-Wahnefried W. Diet quality of cancer survivors and noncancer individuals: Results from a national survey. Cancer 2015; 121 : 4212-21. doi: 10.1002/cncr.29488

40. Hawkins NA, Berkowitz Z, Rodríguez JL. Awareness of Dietary Alcohol Guidelines among colorectal cancer survivors. Am J Prev Med 2015; 49: S509-17. doi: 10.1016/j. amepre.2015.08.012

41. Bagnardi V, Rota M, Botteri E, Tramacere I, Islami F, Fedirko V. et al. Light alcohol drinking and cancer: a meta-analysis. Ann Oncol 2013; 24: 301-8. doi: 10.1093/annonc/ mds 337 
42. Flatt SW, Thomson CA, Gold EB, Natarajan L, Rock CL, Al-Delaimy WK, et al. Low to moderate alcohol intake is not associated with increased mortality after breast cancer. Cancer Epidemiol Biomarkers Prev 2010; 19: 681-8. doi: 10.1158/1055-9965.EPI-090927

43. James EL, Stacey FG, Chapman K, Boyes AW, Burrows T, Girgis A, et al. Impact of a nutrition and physical activity intervention (ENRICH: Exercise and Nutrition Routine Improving Cancer Health) on health behaviors of cancer survivors and carers: a pragmatic randomized controlled trial. BMC Cancer 2015; 15: 710. doi: 10.1186/s12885-0151775-y.

44. Ibfelt E, Rottman N, Kjaer T, Høybye MT, Ross L, Frederiksen K, et al. No change in health behavior, BMI or self-rated health after a psychosocial cancer rehabilitation: Results of a randomized trial. Acta Oncol 2011; 50: 289-98. doi: 10.3109/0284186X.2010.531761.

45. DeNysschen C, Brown JK, Baker M, Wilding G, Tetewsky S, Cho MH, et al. Healthy life style behaviors of breast cancer survivors. Clin Nurs Res 2015; 24: 504-25. doi: $10.1177 / 1054773814553298$

46. Hawkes AL, Chambers SK, Pakenham KI, Patrao TA, Baade PD, Lynch BM, et al. Effects of a telephone-delivered multiple health behavior change information (CanChange) on health and behavioral outcomes in survivors of colorectal cancer: a randomized controlled trial. J Clin Oncol 2013; 31: 2113-21. doi: 10.1200/JCO.2012.45.5873.

47. Fazzino TL, Fleming K, Befort C. Alcohol intake among breast cancer survivors: Change in alcohol use during a wright management intervention. JMIR Cancer 2016; 2(2): e15. doi: 10.2196/cancer.6295

48. Garcia-Torres F, Alós FJ, Pérez-Dueñas C, Moriana Elvira JA. Guía de tratamientos psicológicos eficaces en psicooncología. Madrid: Pirámide, 2016.

49. Witkiewitz K, Marlatt A. Behavioral therapy across the spectrum. Alcohol Res Health 2011; 33: 313-9.

50. National Cancer Institute [Internet]. 27 de Marzo de 2017. Usual Dietary intakes: NHANES Food Frequency Questionnaire (FFQ); [Acceso el 11 de Julio de 2017]. Disponible en: https://epi.grants.cancer.gov/diet/usualintakes/ffq.html 